\title{
Flicker and fusion threshold interdependency
}

\author{
FELIX E. GOODSON, KENNETH S. WAGONER, and MARY B. McCLENDON \\ DePauw University, Greencastle, Indiana 46135
}

\begin{abstract}
This experiment was designed to determine whether a previously established flicker (or fusion) threshold in one eye could be altered by manipulating the light-pulse rate in the other eye. Using an instrument that allowed separate pulse presentations for each eye, both the flicker and fusion thresholds were obtained for one eye (called the target eye). Then the pulse rate was varied in the other eye (called the manipulated eye), in an effort to shift the threshold in the target eye either from flicker to fusion or from fusion to flicker. Although a variety of pulse rates were presented in the manipulated eye near the thresholds of the target eye, there was no significant evidence that the threshold (either fusion or flicker) values were altered. Thus, our view that a flicker-fusion threshold interdependency exists between the two eyes was not supported.
\end{abstract}

Many different types of interocular transfer effect have been reported. These range from the transfer of adaptation to prismatic distortion in humans (Pick, Hay, \& Willoughby, 1966) to the transfer of simultaneous discriminations in the pigeon (Green, Brecha, \& Gazzaniga, 1978). And even though the two eyes can be light and dark adapted independently, it has been reported (Lansford \& Baker, 1969) that the presentation of light to the left eye as the right eye is being light adapted will subsequently accelerate dark adaptation in the right eye. When these and many other findings supporting ocular interdependence were coupled with Hering's (1868) statement (sometimes called Hering's law), "The movement of both eyes are to such an extent united with each other that the one will not move independently of the other" (p. 12), it was hypothesized that an ocular interdependence might be obtained for flicker and fusion thresholds.

\section{METHOD}

Subjects

The subjects consisted of 15 undergraduate students at DePauw University. Both female and male subjects were used.

\section{Apparatus}

A flicker-fusion oscillator, produced by the Lafayette Instrument Company, which allowed separate light-pulse presentations for each eye through a binocular eyepiece, was used. Frequencies were manipulated by a dial on a lefteye control unit and another on a right-eye control unit.

\section{Procedure}

Familiarization treatment. Each subject was seated in front of the apparatus and allowed to observe the changes in frequency of light pulses produced by turning the control dials independently for each eye. The subject was then told that the

Reprint requests should be sent to the first author, Department of Psychology, DePauw University, Greencastle, Indiana 46135. flicker threshold is the point at which a steady light beam begins to flicker as the frequency of the light pulses is reduced and the fusion threshold is the point at which a flickering light becomes fused into a steady light as the frequency of light pulses is increased. The subject was then presented examples of these conditions in each eye. The subject was then allowed to manipulate the dials until he or she became adept at using the apparatus and finding both fusion and flicker thresholds for each eye.

Test trials. Each subject was given 20 test trials. Each trial involved two phases: (1) threshold determination for the target eye and (2) an effort to alter these thresholds by presenting light pulses in the manipulated eye. In 10 trials the left eye was the target eye, and in 10 the right eye was the target eye. In five of the trials for each eye, an effort was made to "drive" flicker into fusion, and in the other five, an effort was made to "pull" fusion into flicker. All conditions were altered randomly.

Target-eye threshold determination. The experimenter set the dial at the lowest possible frequency if fusion was to be determined or at the highest possible frequency if flicker was to be determined. The subject was then told to slowly turn the dial either to the left or to the right (again, depending upon whether fusion or flicker thresholds were being determined) and to stop turning the dial and remove his hand from it as quickly as possible, once he had reached the threshold. The experimenter recorded this frequency.

Manipulation phase. Immediately after the target-eye threshold had been determined, the subject was exposed to pulse stimulation in the manipulated eye while the threshold frequencies were continued in the target eye. If flicker was the established condition in the target eye, the dial was set 5 pulses/ sec under the flicker threshold in the manipulated eye. If fusion was the established condition, the frequency in the manipulated eye was set 5 pulses/sec above the fusion threshold of the target eye. The frequency in the manipulated eye was then gradually reduced by the experimenter in an effort to "pull" the fusion in the target eye into flicker, or it was gradually increased in an effort to "drive" the flicker in the target eye into fusion. In all cases, the frequencies in the manipulated eye were changed until the threshold values in the target eye were exceeded. During each manipulation phase, the subject was instructed to attend to the condition in the target eye and to manipulate the dial for that eye, if such an adjustment was necessary, in order to retain or regain the condition (whether fusion or flicker) that was reached in the threshold determination phase. After this adjustment had been made, the subject was told to remove his hand from the dial and the experimenter recorded the frequency. 


\section{RESULTS}

The mean value for the 10 test scores under each of the test conditions was obtained (the data for the two eyes were combined) for both before manipulation and after manipulation. An analysis of variance was used to determine whether there was a significant shift in thresholds, whether flicker or fusion, between the threshold determination and manipulation conditions. The data for fusion yielded an F of .04, and that for flicker, an $F$ of .02 , with 4.20 required for significance in both cases.

Thus, our hypothesis that a flicker-fusion threshold exists between the two eyes was not supported. Since some of the subjects gave subjective reports that a shift in the predicted direction did take place and since other studies have suggested interocular interdependency, the entire experiment was replicated using 20 subjects: 10 high school seniors and 10 college undergrad- uates. In this study, the frequencies in the manipulated eye were changed until the threshold values for the target eye were exceeded by the entire range of the apparatus, which averaged 15 light pulses/sec. But, once again, the results were negative; the Fs obtained did not approach significance.

\section{REFERENCES}

Green, L., Brecha, N., \& Gazzaniga, M. Interocular transfer of simultaneous but not successive discriminations in the pigeon. Animal Learning \& Behavior, 1978, 6, 261-264.

Hering, E. Die Lehre vom Binocularen Schen. Leipzig: Engelman, 1868.

LANSFond, T. G., \& BAKER, H. D. Dark adaptation: An interocular light-adaptation effect. Science, 1969, 164, 1307-1309.

Pick, H. L., JR., HAy, J. C., \& Willoughby, R. H. Interocular transfer of adaptation to prismatic distortion. Perceptual and Motor Skills, 1966, 23, 131-135.

(Received for publication January 11, 1982.) 